Referencia para citar este artículo: Bernal-Ruiz, F., Rodríguez-Vera, M., González-Campos, J., \& Torres-Álvarez, A. (2018). Competencias parentales que favorecen el desarrollo de funciones ejecutivas en escolares. Revista Latinoamericana de Ciencias Sociales, Niñez y Juventud, 16(1), 163-176. doi:10.11600/1692715x.16109

\title{
Competencias parentales que favorecen el desarrollo de funciones ejecutivas en escolares*
}

\author{
FRANCISCA BERNAL-RUIZ ** \\ Profesora Universidad de Playa Ancha, Chile. \\ MONTSERRAT RODRÍGUEZ-VERA *** \\ Coordinadora acompañamiento, Programa Pace, Universidad de Playa Ancha, Chile. \\ JoSÉ GONZÁLEZ-CAMPOS ${ }^{* * * *}$ \\ Profesor Universidad de Playa Ancha, Chile. \\ ALEXIS TORRES-ÁLVAREZ $Z^{* * * * *}$ \\ Ingeniero Informático, Universidad de Playa Ancha, Chile.
}

- Resumen (analítico): dada la relevancia del desarrollo y estimulación temprana de las Funciones Ejecutivas (FE) en escolares, nuestro objetivo en esta investigación fue determinar si existe relación estadísticamente significativa entre las Competencias Parentales $(C P)$ de 31 padres y madres de escolares de segundo básico de Chile, y las FE y Rendimiento Académico (RA) de estos. Los instrumentos utilizados fueron la Escala de Parentalidad Positiva e2p y el Test de Evaluación Neuropsicológica Infantil Teni. Los resultados evidenciaron que los padres y madres que tienen más desarrolladas las CP protectoras, reflexivas y formativas, tienen hijos o hijas con mayor desarrollo de las FE. Respecto a la relación entre las $C P$ y el $R A$, la encontramos solo en matemáticas. Concluimos que existen $C P$ que se relacionan con el desarrollo de FE y con el RA de los escolares, especialmente en matemáticas.

Palabras clave: relación padres/madres-hijos/hijas, procesos cognitivos, rendimiento escolar (Tesauro de Ciencias Sociales de la Unesco).

\section{Parental competences that favor the development of executive functions in schoolchildren}

- Abstract (analytical): Given the relevance of development and the early stimulation of Executive Functions (EF) among school children, the objective of this study was to determine if there is a statistically significant relationship between the Parenting Competencies (PC) of 31 parents of second-grade schoolchildren in Chile and their EF and Academic Performance (AP). The instruments

Este artículo de investigación científica y tecnológica cuya área de conocimiento es la Psicología, subárea de Psicología Cognitiva y Educacional, está basado en los resultados de la investigación "Prácticas parentales que favorecen el desarrollo de funciones ejecutivas en escolares de segundo año básico", realizada en una Escuela pública de la comuna de Valparaíso, Chile, entre los meses de mayo y diciembre de 2015, con la financiación del Convenio de Desempeño CD/PMI UPA 1203 de la Universidad de Playa Ancha (Upla).

** Doctora en Psicología, Universidad de Oviedo (España), Magíster en Pedagogía Universitaria, Upla (Chile), Psicóloga, Universidad de Valparaíso (Chile). Docente Facultad de Educación Upla. Orcid: 0000-0001-6973-2443. Índice H5: 3. Correo electrónico: fbernal@upla.cl

*** Máster en Neuropsicología, Universidad de Salamanca (España), Magíster en Psicología Social, Universidad de Talca (Chile), Psicóloga, Universidad de Talca (Chile). Orcid: 0000-0003-0356-8526. Correo electrónico: montserrat.rodriguez@upla.cl

***** Doctor en Estadística, Universidade Estadual de Campinas (Brasil), Magíster en Estadística, Pontificia Universidad Católica de Chile, Profesor de Matemática y Computación, Upla (Chile). Orcid: 0000-0003-4610-6874. Índice H5: 2. Correo electrónico: jgonzalez@upla.cl

***** Ingeniero Informático, Upla (Chile). Orcid: 0000-0003-0100-0689. Correo electrónico: alexis.torres@upla.cl 
used included the Positive Parenting Scale (E2P) and the Neuropsychological Assessment Test (Teni). The results showed that the parents who had developed their protective, reflective and formative PC had children with greater EF development. Regarding the correlation between PCs and AP, it was found that this only occurred in the subject area of mathematics. As a conclusion, the research identified that there are PCs that are related to the development of EF and to the AP of schoolchildren, especially in mathematics.

Key words: Parent-child relationship, cognitive processes, academic performance (Social Sciences Unesco Thesaurus).

\section{Competências parentais que favorecem o desenvolvimento das funções executivas em estudantes}

- Resumo (analítico): dada a importância do desenvolvimento e estimulação precoce das funções executivas (FE) na escola, o objetivo deste estudo foi determinar se existe relação estatisticamente significativa entre as competências parentais $(C P)$ de 31 pais de escolas primarias do Chile e suas FE e desempenho acadêmico (RA). Os instrumentos utilizados foram a escada de parentalidade positiva e2p e o teste de Avaliação Neuropsicológica Infantil Teni. Os resultados mostraram que os pais mais desenvolvidos nas CP protetora, reflexiva e formativa, tinham filhos/as com maior desenvolvimento das FE. Quanto à relação entre $C P$ e RA, ele foi encontrado apenas em matemática. Conclui-se que existem CP que relacionam-se com o desenvolvimento das FE e RA de escolares, especialmente em matemática.

Palavras-chave: relações pais-filhos/filhas, processos cognitivos, desempenho acadêmico (Thesaurus de Ciências Sociais da Unesco).

\section{-1. Introducción. -2. Marco Referencial. -3. Metodología. -4. Resultados. -5. Conclusiones y Discusión. -Lista de referencias.}

\section{Introducción}

Actualmente, un tema que ha sido ampliamente estudiado es el de las Funciones Ejecutivas (FE), concepto que ha tomado cada vez mayor fuerza por la asociación que de este se hace con las competencias cognitivas y sociales, así como también por su relación con el éxito escolar (Bernal-Ruiz \& Rodríguez-Vera, 2014; Clark, Pritchard, \& Woodward, 2010). Además, investigaciones suizas han demostrado que las FEen la niñezprevén eldesempeñoacadémicoen matemáticas y lectoescritura, añadiendo ventaja a los niños y niñas que han sido estimulados precozmente (Röthlisberger, Neuenschwander, Cimeli, Michel, \& Roebers, 2012). Otras investigaciones en el ámbito anglosajón, como las de Diamond (2012), Diamond y Lee (2011) y Flook, Smalley, Kitil, Galla y Kaiser-Greenland (2010), señalan que la estimulación temprana de las FE influye de manera significativa en el control conductual y en la regulación cognitiva. A nivel latinoamericano aparecen experiencias que relacionan el estilo cognitivo reflexivoimpulsivo y la capacidad de planificación según el riesgo psicosocial (Arán-Filippetti \& Richaud, 2011).

Dado que las FE se desarrollan desde el nacimiento (Diamond, 2013), las etapas tempranas del desarrollo cobran relevancia, especialmente, las que se relacionan con variables como el temperamento del infante y los estilos de crianza de los progenitores (Cervigni, Stelzer, Mazzoni, \& Alvarez, 2012; Hackman \& Farah, 2009). Así, un estilo de crianza caracterizado por afecto y por apego seguro, propicia el desarrollo de habilidades sociales y con ello las relaciones positivas con los pares, y a su vez disminuye la probabilidad de que se presenten problemas conductuales (Eisenberg, 2009; Ensor \& Hughes, 2008). Del mismo modo, la ausencia o excesiva supervisión y protección de los escolares por parte de sus padres y madres, ha sido vinculada a la irrupción de comportamientos inapropiados en 
distintas etapas del desarrollo (Foster, Garber, \& Durlak, 2008).

En otro estudio relacionado con el funcionamiento ejecutivo se encontró relación entre la estimulación verbal entregada por los padres y madres, y el rendimiento de los hijos e hijas en actividades relacionadas con el lenguaje y la resolución de problemas de carácter no verbal (Landry, 2002), concluyendo que la estimulación verbal favorece el desarrollo futuro de destrezas cognitivas superiores.

De acuerdo con estos datos, que confirman la relevancia que tiene el desarrollo y la estimulación temprana de las $\mathrm{FE}$ en los infantes, en la presente investigación buscamos evidencia acerca de la relación entre las competencias parentales (CP), el desarrollo del funcionamiento ejecutivo y el rendimiento académico (RA), de escolares de segundo año básico, con el fin de aportar conocimiento sobre el rol de los padres y madres en el desarrollo de estas funciones psicológicas superiores.

\section{Marco Referencial}

\subsection{Las Funciones Ejecutivas}

En términos generales, las FE incluyen múltiples funciones que ordenan y dirigen la totalidad de las operaciones cognoscitivas y conductuales. Integran la destreza para comenzar una tarea, inhibir conductas, elegir las acciones necesarias para alcanzar objetivos, elaborar estrategias necesarias orientadas a resolver conflictos difíciles, flexibilizar planes, analizar y juzgar el propio comportamiento, y ordenar y dirigir los procesos de memoria (Isquith, Gioia, \& Espy, 2004; Betancur-Caro, Molina, \& Cañizales-Romaña, 2016).

$\mathrm{Si}$ bien no existe consenso sobre la definición de las FE, o claridad conceptual respecto al constructo, esto puede deberse a que las FE dependen de múltiples procesos cognitivos, lo que dificulta determinar cuáles son sus componentes básicos y cómo estos se organizan en el cerebro (Van der Ven, Kroesbergen, Boom, \& Leseman, 2013). Para comprender las FE, en esta investigación nos basamos en la perspectiva genéticocultural de Luria y en el paradigma histórico- cultural de Vygotsky, autores que describen el funcionamiento mental desde una visión biológica, pero también desde una perspectiva social y cultural (Vygotsky, 1978). Y aunque propiamente no utilizan el término FE en su marco conceptual, hacen referencia a destrezas que involucran iniciativa, interés, formulación de objetivos, planeación de actividades y control conductual (Luria, 1976), las que en la actualidad se vinculan al funcionamiento ejecutivo (Lezak, 1982; Miyake, Friedman, Emerson, Witzki, Howerter, \& Wager, 2000).

Bajo este paradigma, las FE son concebidas como funciones mentales superiores autoreguladas, esenciales para el desarrollo y mediadas por el aprendizaje. Uno de los principales fundamentos desde el paradigma histórico-cultural, es que el desarrollo mental de un niño o niña no es simple maduración por instinto natural, sino que ocurre por el proceso objetivo de la comunicación con los individuos adultos (Luria, 1976), por lo que resulta relevante comprender el funcionamiento mental desde sus etapas más tempranas.

Otros autores se refieren a las FE como una familia de procesos mentales top-down, que se requieren para concentrarse y prestar atención, y para cuando no es posible confiar en las respuestas automáticas (Miller \& Cohen, 2001; Diamond, 2013). Por su parte, Zelazo y Müller (2002), señalan que las FE se clasifican en "frías" y "calientes". Las frías se relacionan con tareas que demandan poco control emocional, como la resolución de problemas, y las calientes, con tareas que requieren de reacciones y control emocional.

A pesar de los múltiples conceptos que definen las FE, existe consenso en tres puntos centrales, observados en el modelo de Miyake et al., (2000), quienes consideraron la unidad y separabilidad de estas FE, incluyendo tres factores: la memoria de trabajo, el control inhibitorio, y la flexibilidad cognitiva. La primera se refiere a un componente implicado en la manipulación de la información entrante, su adaptación y manipulación mental. El control inhibitorio o supresión de respuestas prepotentes, es la capacidad para ignorar los estímulos irrelevantes. Finalmente, la flexibilidad cognitiva implica procesos de 
atención en diferentes demandas de tareas, operaciones y juegos mentales (Miyake \& Friedman, 2012). De estos tres puntos a su vez se desprenden el razonamiento de problemas, la resolución de conflictos y la planificación (Collins \& Koechlin, 2012).

En el contexto escolar se necesitan estas habilidades, ya que los niños y niñas deben ser capaces de concentrarse, ignorar distracciones, atender a sus profesores, seguir las reglas de clase, llevarse bien con sus compañeros y compañeras, esperar recompensas y suprimir impulsos de jugar o de agredir a otros. Por lo tanto, no es sorprendente que las FE se relacionen con el éxito escolar y sean vistas como un elemento clave para la preparación para la escuela (Bernal-Ruiz \& RodríguezVera, 2014; Diamond, 2013; Diamond \& Lee, 2011; Blair \& Razza, 2007).

\subsection{Competencias Parentales y su relación con las Funciones Ejecutivas}

Un tema de progresiva preocupación en el área de la neuropsicología infantil, es el análisis del papel que desempeña el contexto social y el aprendizaje en el funcionamiento ejecutivo en la niñez (Vargas-Rubilar \& AránFilippetti, 2014). Así, de todos los contextos sociales de influencia sobre el desarrollo humano, el que ha despertado mayor interés en los investigadores e investigadoras del comportamiento por su indiscutible aporte tanto en el ámbito socioemocional como en el cognitivo, es el contexto familiar (VargasRubilar \& Arán-Filippetti, 2014), cuya función más importante es dar a los hijos e hijas la estimulación adecuada para que se transformen en individuos con las habilidades cognitivas esenciales para interactuar adecuadamente con su ambiente (Barudy \& Dantagnan, 2010).

Múltiples estudios refieren que un vínculo parental apropiado, caracterizado por el cuidado sensible, la protección, la adecuada estimulación y las relaciones marcadas por el afecto y el buen trato, ejerce una influencia positiva en el desarrollo cerebral temprano (Barudy \& Dantagnan, 2010; Siegel, 2007). Tanto el desarrollo físico como el cognitivo y el socioemocional, surgen de las experiencias relacionales del niño o niña con su padre y su madre, principalmente durante los primeros tres años de vida, las que estimulan las conexiones sinápticas en diferentes zonas del cerebro (Barudy \& Dantagnan, 2010); de ahí que la estimulación cognitiva y afectiva recibida precozmente en el hogar y en el ambiente social, tendrían un importante papel en la organización y el funcionamiento cerebral (Vargas-Rubilar \& Arán-Filippetti, 2014).

Existe bastante evidencia científica de la relación entre las prácticas de crianza y las FE de los hijos e hijas (Vargas-Rubilar \& Arán-Filippetti, 2014). Samuelson, Krueger y Wilson (2012) encontraron que las prácticas parentales positivas se asocian con mejores rendimientos en actividades de planificación y resolución de problemas en los niños y niñas. Del mismo modo, Schroeder y Kelley (2010), hallaron asociaciones significativas entre la estructura familiar, la protección y los límites determinados por los padres y madres, y el funcionamiento ejecutivo de los niños y niñas, específicamente en relación con la planificación, la organización, la memoria de trabajo, la inhibición, la flexibilidad, el monitoreo y el control emocional. Por su parte, Olson, Bates y Bayles (1990), encontraron evidencia de una asociación positiva entre las experiencias relacionales cognitivamente estimulantes entre padres y madres con sus hijos e hijas, y la habilidad de estos para postergar la gratificación y controlar su conducta. En esta misma línea, Bernier, Carlson y Whipple (2010) hallaron que las relaciones que establecen los padres y madres con sus descendientes tienen una importante influencia en el desarrollo de habilidades de autorregulación y de control inhibitorio en los niños y niñas. Por su parte, Rhoades, Greenberg, Lanza y Blair (2011), descubrieron que diversos factores de riesgo psicosocial relacionados con la estructura familiar - entre ellos, el ingreso promedio y el grado de instrucción de sus miembros - tienen una influencia negativa en la calidad de las relaciones interpersonales padres/madres-hijos/hijas, lo que a su vez impacta en el funcionamiento ejecutivo de los sujetos infantes. Finalmente, Hughues y Ensor (2009) encontraron que variados factores familiares favorecen el desarrollo de las FE de 
los hijos e hijas, tales como el apoyo y guía parental en la ejecución de tareas orientadas a metas, las prácticas parentales estables y consistentes, y la buena organización familiar, factores que ayudan a los niños y niñas a prever las consecuencias de sus acciones, a establecer objetivos y a resolver distintas situaciones problemáticas.

Las prácticas de crianza de los padres y madres dan cuenta de las competencias parentales (CP) con las que estos cuentan. Estas competencias se definen como "las capacidades prácticas que tienen las madres y los padres para cuidar, proteger y educar a sus hijos, y asegurarles un desarrollo suficientemente sano" (Barudy, \& Dantagnan, 2010, p. 34).

Por su parte, Gómez y Muñoz (2014) las definen como:

La adquisición demostrada $-\mathrm{y}$ posterior desarrollo - de conocimientos y capacidades para conducir el comportamiento parental propio, a través de diversas situaciones de la vida familiar y la crianza y en las distintas dimensiones (física, cognitiva, comunicativa, socioemocional) del desarrollo del niño o niña, con la finalidad última de garantizar su bienestar y el ejercicio pleno de sus derechos humanos (p. 20).

Según estos mismos autores, las CP cubren cuatro áreas: vínculo, formación, protección y reflexión. Las competencias vinculares incluyen las prácticas parentales orientadas a la promoción de un apego seguro y un desarrollo socioafectivo apropiado en los hijos e hijas. Por su parte, las competencias formativas integran las prácticas de crianza orientadas a propiciar el desarrollo cognitivo y social de los sujetos infantes. A su vez, las competencias protectoras incluyen las prácticas parentales orientadas a brindar protección y cuidado a los hijos e hijas, asegurando su integridad tanto física como emocional. Y finalmente, las competencias reflexivas incluyen las prácticas de crianza orientadas al razonamiento sobre el propio estilo parental, la revisión sistemática de las prácticas asociadas a este y la evaluación del desarrollo de los hijos e hijas, con el propósito de retroalimentar las otras competencias parentales (Gómez \& Muñoz, 2014).
Tal como quedó evidenciado anteriormente, las competencias parentales positivas se relacionan con un mejor funcionamiento ejecutivo y un desempeño más satisfactorio de los niños y niñas en tareas de planificación y resolución de problemas (Vargas-Rubilar \& Arán-Filippetti, 2014); de allí la importancia de conocerlas y propiciarlas en el entorno familiar.

\subsection{Funciones Ejecutivas y Rendimiento Académico}

El análisis de los factores que determinan el rendimiento académico (RA) ha sido constantemente de especial interés para los investigadores e investigadoras; sin embargo, las variables que durante muchos años han utilizado para intentar explicarlo y predecirlo, se centran principalmente en las relacionadas con la inteligencia, el estrato socioeconómico, la motivación y el tipo de enseñanza (Diamond, 2013; Zambrano, 2013). Esto evidencia la urgente necesidad de investigar nuevas variables que expliquen el desempeño de los niños y niñas en el ámbito escolar. Y en este contexto, las FE han despertado un notable interés para los investigadores e investigadoras durante los últimos tiempos.

Actualmente es posible encontrar múltiples investigaciones (Clair-Thompson \& Gathercole, 2006; McClelland et al., 2007) que han mostrado que las habilidades ejecutivas favorecen el rendimiento académico (RA). Entre ellas, la de Korzeniowski (2011), quien en su investigación respecto a la evolución de las FE y su relación con el aprendizaje escolar, hace referencia a la existencia de tres períodos sensibles en el desarrollo de las FE, en los cuales está acrecentada la neuroplasticidad cerebral, lo que posibilita que el funcionamiento ejecutivo sea ampliamente estimulado por las experiencias favorables. Estos períodos van de los 6 a los 8 , de los 10 a los 12 y de $\operatorname{los} 15$ a los 19 años de edad, etapas que coinciden con la experiencia de aprendizaje escolar.

En esta misma línea de investigación se hallan los estudios de Arán-Filippetti (2011), quien concluyó que el funcionamiento ejecutivo se ve favorecido con el ingreso al sistema escolar; de igual manera, Diamond y Lee 
(2011) plantearon que un buen funcionamiento ejecutivo en la infancia se relaciona con posteriores resultados académicos y éxito escolar.

Del mismo modo, el desempeño académico exitoso de los niños y niñas en edad escolar ha sido relacionado en forma positiva con competencias parentales equilibradas y receptivas (Vargas-Rubilar \& Arán-Filippetti, 2014).

A partir de la evidencia hasta aquí referida, podemos concluir que tanto el entorno como la experiencia social, cognitiva y emocional que han tenido los niños y niñas, influyen en mayor o menor medida en el funcionamiento ejecutivo, y consecuencialmente en el RA de estos. Por lo mismo, nuestro propósito en este estudio ha sido el de encontrar evidencia que contribuya al conocimiento y la comprensión de cuáles son las CP que promueven el desarrollo de las FE e inciden en el RA de los sujetos escolares de la muestra.

\section{Hipótesis}

- Existen diferencias estadísticamente significativas $(\mathrm{p}=0,05)$ entre las competencias parentales de los padres y madres o individuos cuidadores de escolares de segundo básico de la Escuela Naciones Unidad (una escuela pública) de Valparaíso, y las funciones ejecutivas de estos sujetos escolares.

- Existen diferencias estadísticamente significativas $\quad(p=0,05)$ entre las competencias parentales de los padres y madres o individuos cuidadores de escolares de segundo básico de la Escuela Naciones Unidad (una escuela pública) de Valparaíso y el rendimiento académico de estos sujetos escolares.

- Existe relación estadísticamente significativa $(p=0,05)$ entre las funciones ejecutivas y el rendimiento académico de escolares de segundo básico de la Escuela Naciones Unidad (una escuela pública) de Valparaíso.

\section{Objetivo General}

- Determinar si existen diferencias estadísticamente significativas $(\mathrm{p}=0,05)$ entre las competencias parentales de los padres y madres o individuos cuidadores de escolares de segundo básico de la Escuela Naciones Unidad (una escuela pública) de Valparaíso y las funciones ejecutivas y el rendimiento académico de estos sujetos escolares.

\section{Objetivos Específicos}

- Determinar si existen diferencias estadísticamente significativas $(p=0,05)$ entre las competencias parentales de los padres y madres o individuos cuidadores $\mathrm{y}$ las funciones ejecutivas de los sujetos escolares de segundo básico de la Escuela Naciones Unidad (una escuela pública) de Valparaíso.

- Determinar si existen diferencias estadísticamente significativas $(p=0,05)$ entre las competencias parentales de los padres y madres o individuos cuidadores y el rendimiento académico de los sujetos escolares de segundo básico de la Escuela Naciones Unidad (una escuela pública) de Valparaíso.

- Establecer si existe relación estadísticamente significativa $(\mathrm{p}=0,05)$ entre las funciones ejecutivas $\mathrm{y}$ el rendimiento académico de los sujetos escolares de segundo básico de la Escuela Naciones Unidad (una escuela pública) de Valparaíso.

\section{Metodología}

\subsection{Tipo y Diseño de investigación}

El tipo de investigación es descriptivo correlacional, con un diseño no experimental, transeccional.

Las variables de estudio presentan un estatus métrico cuantitativo:

VI: Competencias parentales.

VD: Funciones Ejecutivas y Rendimiento Académico.

\subsection{Participantes}

La muestra estuvo conformada por 31 padres/madres o individuos cuidadores, de los cuales $29(93 \%)$ eran mujeres y $2(7 \%)$ eran 
hombres, y sus respectivos hijos e hijas, de los cuales 18 eran niños (58\%) y 13 niñas (42\%), quienes cursaban segundo básico en la Escuela Naciones Unidad (una escuela pública) de la comuna de Valparaíso, Chile, en el año 2015.

\subsection{Instrumentos}

Empleamos dos instrumentos, uno destinado a los sujetos escolares y otro para evaluar a los padres y madres o individuos cuidadores.

Para evaluar las FE de los sujetos escolares empleamos el Test de Evaluación Neuropsicológica Infantil Teni, específicamente las cuatro subpruebas que evalúan las FE. Este instrumento fue creado por Tenorio, Arango, Aparicio, Benavente, Thibaut y Rosas (2012), investigadores del Centro de Desarrollo de Tecnologías de Inclusión de la Pontificia Universidad Católica de Chile, Cedeti UC. Está compuesto por ocho subpruebas independientes entre sí, las cuales se presentan como juegos en una Tablet, para evaluar cinco funciones cognitivas: atención, desarrollo viso-espacial, sistemas de memoria, lenguaje y FE. Se puede aplicar a niños desde los 3:0 hasta los 9:11 años.

Las subpruebas del Teni que evalúan las FE corresponden a:

- "Torpo el Topo Torpe", que mide Memoria de trabajo, y en la cual un topo aparece y desaparece en distintos túneles distribuidos en una grilla en la pantalla; el niño o niña debe observar estas apariciones, y posteriormente, tras escuchar una campana, repetirlas tocando en la pantalla táctil los túneles en el orden en que apareció el topo. La complejidad aumenta cada dos respuestas correctas. La variable final analizada en esta subprueba es la suma de aciertos durante los ensayos realizados.

- "La Granja” evalúa la habilidad del niño o niña para seguir patrones y completar series lógicas. En ella el sujeto escolar debe completar una serie que se le presenta, eligiendo una de tres opciones. Las series van aumentando de complejidad al avanzar la prueba, la que se suspende luego de cuatro respuestas incorrectas consecutivas.
- “Bzz! - Inhibición” mide la capacidad que tiene el niño o niña para autorregular su conducta, y las habilidades con las que cuenta para lograr retrasar la gratificación. En esta subprueba aparecen en la pantalla táctil una serie de moscas volando azarosamente, las cuales debe matar el sujeto participante presionándolas con su dedo; esto durante 1 minuto, luego de lo cual se le indica que el evaluador o evaluadora se va a ausentar, y que durante ese tiempo (5 minutos) no debe presionar la pantalla para matar las moscas. El resultado de esta subprueba va en directa relación a si el niño o niña inhibió o no la conducta - tocar la pantalla—, y si no la inhibió, a la latencia hasta que tocó la pantalla.

- "Trini y Agu" mide la habilidad del niño o niña para comprender y predecir la conducta de otras personas. En ella, el guión de Baron-Cohen, Leslie y Frith (1985) se presenta en formato de comic y se formulan tres preguntas de creencias. El resultado de esta subprueba se obtiene a partir del número de respuestas correctas.

El Teni ha sido estandarizado en Chile, manifestando niveles de confiabilidad en rangos de "bueno" a "excelente". Está orientado a la norma y ha sido diseñado con una concepción modular funcional de la cognición (Tenorio et al., 2012). Su aplicación dura aproximadamente 30 minutos.

Para evaluar las CP de los padres/madres o sujetos cuidadores, utilizamos la Escala de Parentalidad Positiva e2p, creada por Gómez y Muñoz (2014), que identifica las CP que un individuo adulto responsable de la crianza de un niño o niña usa al relacionarse con él o ella.

El e2p es un cuestionario auto administrado que consta de 54 ítems que describen prácticas habituales de crianza en cuatro áreas: vínculo, formación, protección y reflexión. De estas áreas surgen sus cuatro subescalas: Competencias Vinculares (14ítems), Competencias Formativas (12 ítems), Competencias Protectoras (17 ítems) y Competencias Reflexivas (11 ítems). Para responder, se debe escoger entre 4 opciones: Casi nunca, A veces, Casi siempre y Siempre. Su consistencia interna está en los rangos de 
"bueno" a "excelente". Su aplicación es de aproximadamente 20 minutos.

Para obtener el puntaje de las subescalas se debe sumar los puntajes de los ítems asociados a ellas, y para la obtención del puntaje de la escala de Competencia Parental Total se suman los puntajes de las 4 subescalas. Con este puntaje se obtiene el percentil del sujeto evaluado. Para interpretar los resultados, se distinguen 3 rangos o zonas de CP; según dicho percentil, la "zona de riesgo" incluye los percentiles 10 y 20; la "zona de monitoreo" los percentiles 30 y 40 y la "zona óptima" los percentiles 50 o superior.

Finalmente, para la evaluación del RA de los sujetos escolares de la muestra, utilizamos las calificaciones consignadas por sus docentes en el libro de clases. Consideramos el promedio de notas general y el de los subsectores de matemática y lenguaje, tanto del primero como del segundo semestre del año escolar 2015.

\subsection{Procedimiento}

Esta investigación la adelantamos en una Escuela pública de la comuna de Valparaíso, Chile. Participamos de dos reuniones de apoderados del segundo básico para explicar los objetivos de la investigación, firmar el consentimiento informado y aplicar el Cuestionario de Parentalidad positiva e2p a los padres/madres o sujetos cuidadores asistentes.

Luego procedimos a evaluar las FE de los estudiantes y las estudiantes de segundo básico, en la biblioteca del propio establecimiento y en horario de clases. Este proceso duró alrededor de dos semanas.

Respecto a la estimación del RA de los sujetos escolares de la muestra, en el mes de diciembre, al finalizar el segundo semestre del año 2015, solicitamos a la Jefa técnica del establecimiento los promedios de notas de las asignaturas de matemática, lenguaje, y el promedio general de los estudiantes.

Para analizar los datos utilizamos la comparación de medias (t-student) y correlación de Pearson. A su vez, aplicamos el test F-Snedecor para el estudio de dependencia entre las variables. Para analizar los datos, empleamos el software $\mathrm{R}$ project 3.3.2. La mayoría de las pruebas fueron realizadas a un
$5 \%$ de significancia, utilizando como criterio de decisión el p-valor.

\section{Resultados}

En primer lugar, y con el fin de comprobar si las cuatro CP evaluadas por la escala $\mathrm{e} 2 \mathrm{p}$ eran independientes entre sí, determinamos la correlación de Pearson, y encontramos que las cuatro competencias eran variables linealmente independientes, con niveles de explicación que no superan el $1,5 \%$, a saber: competencias vinculares $\quad(p$-valor $=0,71), \quad$ competencias formativas $\quad(p$-valor $=0,50), \quad$ competencias protectoras $(\mathrm{p}$-valor $=0,52)$ y competencias reflexivas ( $\mathrm{p}$ valor $=0,78)$.

Para determinar la existencia de diferencias estadísticamente significativas $(p=0,05)$ entre las $\mathrm{CP}$ de los padres/madres o sujetos cuidadores y las FE de los sujetos escolares de la muestra, realizamos un análisis en función del desarrollo o no de cada una de las CP, mediante la comparación de medias (prueba t-student). Así, al relacionar las cuatro $\mathrm{CP}$ en conjunto con las $\mathrm{FE}$, no observamos diferencias significativas al $95 \%$ de confianza, y obtuvimos un p-valor de 0,31 al 95\% de confianza. Por lo mismo, y para enriquecer el análisis, realizamos un contraste de medias considerando las cuatro $\mathrm{CP}$ por separado y la relación de cada una con las subpruebas del Teni que evalúan las FE, hallando lo siguiente:

Encontramos diferencias significativas en las puntuaciones de las FE de los sujetos escolares, con un $95 \%$ de confianza, a favor de los padres/madres o sujetos cuidadores que tenían desarrolladas las competencias protectoras o las reflexivas con $\mathrm{p}$-valores de 0,02 y 0,04 respectivamente. En el caso de las competencias vinculares y las formativas, al 95\% de confianza, no evidenciamos diferencias significativas entre tenerlas o no tenerlas desarrolladas y el funcionamiento ejecutivo de los hijos e hijas; sin embargo, en el caso de las competencias formativas, al flexibilizar la significancia a un $10 \%$ sí observamos cambios significativos en las puntuaciones de las FE de los hijos e hijas a favor de los padres/madres o sujetos cuidadores que las tenían desarrolladas $(\mathrm{p}$-valor=0,06). 
El desempeño de los sujetos escolares en la subprueba Topo del Teni, presenta diferencias significativas ( $p$ - valor $=0,06$ ) con un $90 \%$ de confianza, en función del desarrollo de las competencias formativas que sus padres/ cuidadores tengan. En otras palabras, a mayor desarrollo de las competencias parentales formativas —orientadas a propiciar el desarrollo, socialización y aprendizaje de los individuos infantes-, mejor desempeño tienen sus hijos e hijas en la subprueba Topo, que evalúa la memoria de trabajo. No ocurre lo mismo al relacionar las competencias formativas con las subpruebas la Granja, Bzz! y Trini y Agu, en las que no observamos diferencias significativas con p-valores de 0,23 , 0,83 y 0,83 , respectivamente.

Tanto en las competencias protectoras como en las reflexivas tampoco encontramos diferencias significativas al relacionarlas con las subpruebas Topo, la Granja, Bzz! y Trini y Agu, al 95\% de confianza, con p-valores de 0,$11 ; 0,90 ; 0,49$ y 0,39 para las competencias protectoras y 0,$42 ; 0,36 ; 0,56$ y 0,11 para las competencias reflexivas. Sin embargo, ambas competencias sí evidenciaron diferencias significativas al relacionarlas con las $\mathrm{FE}$ en general.

Ahora bien, para determinar la existencia de diferencias estadísticamente significativas $(p=0,05)$ entre las CP y el RA, aplicamos un test F-Snedecor, donde obtuvimos un p-valor de 0,81, lo que nos permite concluir que las $\mathrm{CP}$ y el RA son variables linealmente independientes. No obstante, como no encontramos correlación entre estas dos variables, y para enriquecer el análisis, posteriormente consideramos las Competencias Parentales Óptimas, que como lo señalamos anteriormente, surgen a partir de la ubicación de los padres/madres o sujetos cuidadores en la "zona óptima" de la Escala e2p, en función de su puntaje obtenido (Gómez \& Muñoz, 2014). Lo mismo para la variable $\mathrm{RA}$, en la cual establecimos un umbral de categorización (nota 5,0), para determinar el Rendimiento Académico Matemática Óptimo (Ramo) y el Rendimiento Académico Lenguaje Óptimo (Ralo).

De acuerdo con esta categorización, y para relacionar Competencias Parentales Óptimas y Rendimiento Académico Óptimo tanto en matemática como en lenguaje, aplicamos un test F-Snedecor al 90\% de confianza, arrojando como resultado para el Ramo un p-valor de 0,08 , lo que evidenció que estas variables se relacionan estadísticamente, es decir, son linealmente dependientes. Y para el Ralo, un p-valor de 0,45 , evidenciando independencia lineal entre dichas variables.

Por su parte, al relacionar el Ramo con cada una de las cuatro CP, aplicando el test F-Snedecor al 90\% de confianza, obtuvimos que existe relación estadísticamente significativa solo con las competencias protectoras y las reflexivas con p-valores de 0,07 y 0,10, respectivamente, en tanto con las competencias vinculares y formativas los p-valores fueron de 0,21 y 0,37 , por lo que asumimos que no hay correlación significativa entre dichas variables.

El mismo análisis realizamos con el Ralo al 90\% de confianza, y solo encontramos relación estadística con las competencias reflexivas con un p-valor de 0,06 , lo que da cuenta de que son linealmente dependientes. Con las otras competencias parentales no observamos relaciones significativas.

Finalmente, y con el fin de determinar si existía relación estadísticamente significativa entre las FE y el RA general y el de matemática y lenguaje, aplicamos un test F-Snedecor al $5 \%$ de significancia, encontrando que estas tres variables son linealmente independientes, con p-valores de 0,99, 0,83 y 0,68, respectivamente.

Cabe destacar que, si bien no hallamos relación entre las FE generales y el RA, al relacionar este último con las subpruebas de FE del Teni, encontramos que el $17 \%$ de la variabilidad del RA es explicado por la subprueba Bzz!, que evalúa la autorregulación conductual, lo que evidencia una relación estadísticamente significativa entre ellas, con un p-valor de 0,02 . Con las otras subpruebas no observamos relación estadística (Topo $\mathrm{p}$-valor $=0,85$; la Granja $\mathrm{p}$ - valor $=0,21$ y Trini y Agu p-valor $=0,10)$.

Similar resultado se obtiene al relacionar las FE con el RA en Matemática (RAM) y el RA en Lenguaje (RAL), donde se evidenció que la subprueba Bzz! explica la variabilidad de RAM en un $14 \%$ (p-valor $=0,03$ ) y de RAL en un $25 \%$ 
( $\mathrm{p}$-valor $=0,00)$, lo que traduce en evidencia una relación estadísticamente significativa entre estas variables. En otras palabras, mientras más autorregulado es el niño o niña, mejor es su RA general y su rendimiento en matemática y lenguaje. No ocurre lo mismo con las otras subpruebas.

\section{Conclusiones y Discusión}

Los resultados de la presente investigación nos permiten confirmar la hipótesis inicial referente a la existencia de $\mathrm{CP}$ que favorecen el funcionamiento ejecutivo de los sujetos escolares de la muestra. Esto, en función de que los resultados evidencian que los padres/madres o individuos cuidadores de los sujetos escolares que tenían más desarrolladas las competencias protectoras, reflexivas y formativas, tenían hijos e hijas con mayor desarrollo de las FE. Este hallazgo es congruente con lo que plantea Bornstein (2012) respecto a que las prácticas parentales orientadas al cuidado y protección de los niños y niñas, generan un ambiente de estimulación y libertad de exploración física que favorece el funcionamiento cognitivo de estos.

Por su parte, las competencias parentales reflexivas favorecen el proceso de metaparentalidad (Nicholson, Howard, \& Borkowski, 2008), incidiendo positivamente en el desarrollo de las FE de los hijos e hijas, ya que propician anticipar escenarios, monitorear influencias sobre el propio estilo parental y su historia, reflexionar sobre el ejercicio parental actual y sobre la calidad de la relación con los hijos e hijas. Estos elementos se relacionan con el control que tienen los padres y madres de su conducta, y el traspaso de estos elementos a sus hijos e hijas.

El hallazgo de la relación entre las competencias parentales formativas y las FE, es afín a lo que plantea Aguirre-Dávila (2015), respecto a que existe asociación entre el funcionamiento ejecutivo y las conductas prosociales, entendidas como las acciones dirigidas a ayudar a otros, que son sistemáticas y permanentes, y que en la mayoría de los casos son incentivadas por la familia.
Un aspecto interesante es que no encontramos asociación entre las FE y las competencias parentales vinculares. Esto, posiblemente porque estas $\mathrm{CP}$ están más relacionadas con prácticas orientadas a promover el desarrollo socioemocional de los hijos e hijas, las que incluyen contacto físico y visual frecuente e intercambios verbales más positivos y afectivos con sus descendientes (Bornstein, 2012) que con las habilidades cognitivas asociadas al funcionamiento ejecutivo como tal.

Otro hallazgo que merece la pena analizar, es la relación estadísticamente significativa entre las Competencias Parentales Óptimas y el Rendimiento Académico Matemático Óptimo (Ramo). Este resultado es congruente con lo planteado por Epstein (2013), respecto a que la percepción de los padres y madres sobre las habilidades de sus hijos e hijas en el área matemática, está altamente relacionada con los resultados académicos que estos tengan en dicha área. También con los estudios que afirman que tanto las relaciones parentales positivas, caracterizadas por la cercanía, los buenos tratos, el respeto, la aceptación, la comunicación y la empatía, como el involucramiento familiar en las actividades escolares de los hijos e hijas, tienen un efecto positivo en el desempeño y éxito escolar de estos (Weiss, 2014; Alcalay, Flores, Milicic, Portales, \& Torretti, 2003).

Por otra parte, es importante destacar que, si bien en esta investigación no encontramos evidencia respecto a la asociación entre FE y $\mathrm{RA}$, sí observamos que existen CP que favorecen la regulación del individuo estudiante, y que estarían relacionadas con rendimientos óptimos en matemática y lenguaje; hallazgo que relacionamos con lo planteado por Bol, Riggs, Hacker y Nunnery (2010), respecto a que los padres y madres que trabajan en la planificación, en la flexibilidad cognitiva y en la regulación de la conducta - elementos propios del funcionamiento ejecutivo-, aportan a la mejora del RA de sus hijos e hijas. No obstante, es un tema que debe seguir siendo investigado.

A modo de sugerencias, cabe señalar que, si bien en este trabajo destacamos la relevancia que tienen las $\mathrm{CP}$ en el desarrollo de las FE y el RA de escolares, se necesita 
ahondar en el conocimiento sobre cuáles son las prácticas parentales específicas que inciden mayoritariamente en la evolución de estas funciones y cómo interactúan en distintos contextos; por lo que es necesario seguir realizando estudios que permitan indagar la relación entre las FE, el RA y las $\mathrm{CP}$, incrementando el tamaño muestral, así como también integrando escolares de distintos estratos sociales y muestras que sean más equitativas en cuanto a sexo.

También sería interesante, en futuros estudios, contar con un instrumento estandarizado de evaluación del RA, que mida principalmente conocimientos de lenguaje y matemática, como una herramienta que otorgue mayor objetividad a la medición, en el entendido de que las calificaciones escolares no siempre son expresión objetiva del rendimiento, ya que pueden verse afectadas por factores subjetivos y sociales no siempre controlables por los investigadores e investigadoras (Diamond, 2013, Zambrano, 2013).

En cualquier caso, y a pesar de la necesidad de mejorar ciertos aspectos, con el presente estudio aportamos datos objetivos acerca de la relación entre las $\mathrm{CP}$ y el funcionamiento ejecutivo y RA de escolares, lo que sirve no solo de insumo para nuevas investigaciones en torno al tema, sino también como evidencia para el diseño de programas de apoyo y acompañamiento para padres y madres, que promuevan la adquisición y/o fortalecimiento de CP positivas, las que favorecen el desarrollo socioemocional y cognitivo de los hijos e hijas; y que, al mismo tiempo, los capacite para estimular tempranamente las FE de estos, lo que a su vez favorecería su aprendizaje escolar y disminuiría su fracaso.

Por último, destacamos la importancia de impulsar investigaciones en esta línea, que sirvan de referencia en la materialización de políticas públicas de cuidado y protección infantil y de apoyo a la parentalidad positiva.

\section{Lista de referencias}

Aguirre-Dávila, E. (2015). Prácticas de crianza, temperamento y comportamiento prosocial de estudiantes de educación básica. Revista
Latinoamericana de Ciencias Sociales, Niñez y Juventud, 13(1), 223-243. doi:10. 11600/1692715x.13113100314

Alcalay, L., Flores, A., Milicic, N., Portales, J., \& Torretti, A. (2003). Familia y escuela: ¿una alianza posible? Una mirada desde la perspectiva de los estudiantes. Revista Psykhe, 12(2), 101-110.

Arán-Filippetti, V. (2011). Funciones Ejecutivas en niños escolarizados: efectos de la edad y del estrato socioeconómico. Universitas Psychologica, 29(1), 98-113.

Arán-Filippetti, V., \& Richaud, M. (2011). Efectos de un programa de intervención para aumentar la reflexividad y la planificación en un ámbito escolar de alto riesgo por pobreza. Universitas Psychologica, 10(2), 341-354.

Baron-Cohen, S., Leslie, A., \& Frith, U. (1985). Does the autistic child have a "theory of mind"? Cognition, 21(1), 37-46. doi:10.1016/0010-0277(85)90022-8

Barudy, J., \& Dantagnan, M. (2010). Los desafios invisibles de ser padre o madre: Manual de evaluación de las competencias y la resiliencia parental. Barcelona: Gedisa.

Bernal-Ruiz, F., \& Rodríguez-Vera, M. (2014). Estimulación Temprana de las Funciones Ejecutivas en escolares, una revisión actualizada. Revista de Orientación Educacional, 28(53), 15-24.

Bernier, A., Carlson, S., \& Whipple, N. (2010). From external regulation to selfregulation: Early parenting precursors of young children's executive functioning. Child Development, 81(1), 326-339. doi:10.1111/ j.1467-8624.2009.01397.x

Betancur-Caro, M., Molina, D., \& CañizalesRomaña, L. (2016). Entrenamiento cognitivo de las funciones ejecutivas en la edad escolar. Revista Latinoamericana de Ciencias Sociales, Niñez y Juventud, 14(1), 359-368. doi:10.11600/169271 $5 x .14124160615$

Blair, C., \& Razza, R. (2007). Relating effortful control, executive function, and false belief understanding to emerging math and literacy ability in kindergarten. Child Development, 78(2), 647-663. doi:10.1111/ j.1467-8624.2007.01019.x 
Bol, L., Riggs, R., Hacker, D., \& Nunnery, J. (2010). The calibration accuracy of middle school students in math classes. Journal of Research in Education, 21(2), 81-96.

Bornstein, M. (2012). Parenting Infants. En M. H. Bornstein (Ed.), Handbook of parenting. Volume 1: Children and parenting (pp.343). New York: Psychology Press.

Clair-Thompson, H., \& Gathercole, S. (2006). Executive functions and achievements in school: Shifting, updating, inhibition, and working memory. The Quarterly Journal of Experimental Psychology, 59(4), 745759. doi: 10.1080/17470210500162854

Clark, C., Pritchard, E., \& Woodward, J. (2010). Preschool executive functioning abilities predict early mathematics achievement. Developmental Psychology, 46(5), 11-76. doi:10.11144/Javeriana.UPSY13-4.efds

Collins, A., \& Koechlin, E. (2012). Reasoning, learning, and creativity: Frontal lobe function and human decision-making. PLoS Biology, 10(3). doi:10.1371/journal. pbio. 1001293.

Diamond, A. (2012). Activities and programs that improve children's executive functions. Current directions in psychological science, 21(5), 335-341. doi:10.1177/0963721412453722

Diamond, A. (2013). Executive Functions. Annual Review of Psychology, 64, 135-168. Recuperado de: http://dx.doi.org/10.1146/ annurev-psych-113011-143750.

Diamond, A., \& Lee, K. (2011). Interventions shown to aid executive function development in children 4 to 12 years old. Science, 333(6045), 959-964. doi:10.1126/ science. 1204529

Eisenberg, N. (2009). Relations of parenting style to Chinese children's effortful control, ego resilience, and maladjustment. Development and Psychopathology, 21(2), 455-477. Recuperado de: https://doi. org/10.1017/S095457940900025X

Ensor, R., \& Hughes, C. (2008). Content or connectedness? Mother-child talk and early social understanding. Child Development, 79(1), 201-216. doi:10.1111/j.14678624.2007.01120.x
Epstein, J. (2013). Programas efectivos de involucramiento familiar en las escuelas: estudios y prácticas. Santiago de Chile: Fundación CAP.

Flook, L., Smalley, S., Kitil, J., Galla, B., \& Kaiser-Greenland, S. (2010). Effects of mindful awareness practices on executive functions in elementary school children. Journal of Applied School Psychology 26(1), 70-95. Recuperado de: https://doi. org/10.1080/15377900903379125

Foster, C., Garber, J., \& Durlak, J. (2008). Current and past maternal depression, maternal interaction behaviors, and children's externalizing and internalizing symptoms. Journal of Abnormal Child Psychology, 36(4), 527-537. doi:10.1007/ s10802-007-9197-1

Gómez, E., \& Muñoz, M. (2014). Escala de Parentalidad Positiva e2p. Santiago de Chile: Fundación Ideas para la Infancia.

Hackman, D., \& Farah, M. (2009). Socioeconomic status and the developing brain. Trends in Cognitive Sciences, 13(2), 65-73. doi:10.1016/j.tics.2008.11.003

Hughes, C., \& Ensor, R. (2009). How do families help or hinder the emergence of early executive function? En C. Lewis, \& J. I. M. Carpendale (Eds.), New Directions in Child and Adolescent Psychiatry, (123), 35-50. doi:10.1002/cd.234

Isquith, P., Gioia, G., \& Espy, K. (2004). Executive function in preschool children: Examination through everyday behavior. Developmental Neuropsychology, 26(1), 403-422.

Korzeniowski, C. (2011). Desarrollo evolutivo del funcionamiento ejecutivo y su relación con el aprendizaje escolar. Revista de Psicología Universidad Católica de Argentina, 7(13), 7-26.

Landry, S. (2002). The role of early parenting in children's development of executive processes. Developmental Neuropsychology, 21(1), 15-41. doi: $10.1207 /$ S15326942DN2101_2

Lezak, M. D. (1982). The problem of assessing executive functions. International Journal of Psychology, 17, 281-297. 
Luria, A. (1976). The Working Brain: An Introduction to Neuropsychology. Nueva York: Basic Books.

Cervigni, M., Stelzer, F., Mazzoni, C., \& Alvarez, M. A. (2012). Desarrollo de las funciones ejecutivas en niños preescolares. Una revisión de su vínculo con el temperamento y el modo de crianza. Revista Nacional de la Facultad de Psicología de la Universidad Cooperativa de Colombia. 8(15), 128-139. Recuperado de: http:// dx.doi.org/10.1692/issn.1900-3099

McClelland, M., Cameron, C., Connor, C., McDonald, F., Carrie L., Jewkes, A., \& Morrison, F. (2007). Links between behavioral regulation and preschoolers' literacy, vocabulary, and math skills. Developmental Psychology, 43(4), 947959. doi:10.1037/0012-1649.43.4.947

Miller, E., \& Cohen, J. (2001). An integrative theory of prefrontal cortex function. Annual Review of Neuroscience, 24(1), 167-202. doi:10.1146/annurev.neuro.24.1.167

Miyake, A., \& Friedman, N. (2012). The nature and organization of individual differences in executive functions: Four general conclusions. Current Directions in Psychological Science, 21(1), 8-14. doi:10.1177/0963721411429458

Miyake, A., Friedman, N., Emerson, M., Witzki, A., Howerter, A., \& Wager, T. (2000). The unity and diversity of executive functions and their contributions to complex "frontal lobe" tasks: A latent variable analysis. Cognitive Psychology, 41(1), 49100. doi:10.1006/cogp.1999.0734

Nicholson, J., Howard, K., \& Borkowski, J. (2008). Mental models for parenting: Correlates of metaparenting among fathers of young children. Fathering, 6(1), 39. doi:10.3149/fth.0601.39

Olson, S., Bates, J., \& Bayles, K. (1990). Early antecedents of childhood impulsivity: The role of parent-child interaction, cognitive competence, and temperament. Journal of Abnormal Child Psychology, 18(3), 317334. doi: 10.1007/BF00916568

Rhoades, B., Greenberg, M., Lanza, S., \& Blair, C. (2011). Demographic and familial predictors of early executive function development: Contribution of a person-centered perspective. Journal of Experimental Child Psychology, 108(3), 638-662. doi:10.1016/j.jecp.2010.08.004

Röthlisberger, M., Neuenschwander, R., Cimeli, P., Michel, E., \& Roebers, C. (2012). Improving executive functions in 5-and 6-year-olds: Evaluation of a small group intervention in prekindergarten and kindergarten children. Infant and Child Development, 21(4), 411-429. doi:10.1002/ icd. 752

Samuelson, K., Krueger, C., \& Wilson, C. (2012). relationships between maternal emotion regulation, parenting and children's executive functioning in families exposed to intimate partner violence. Journal of Interpersonal Violence, 27(17), 3532-3550. doi: $10.1177 / 0886260512445385$

Schroeder, V., \& Kelley, M. (2010). Family environment and parent-child relationships as related to executive functioning in children. Early Child Development and Care, 180(10), 1285-1298. doi:10.1080/03004430902981512

Siegel, D. (2007). La mente en desarrollo. Cómo interactúan las relaciones y el cerebro para modelar nuestro ser. Bilbao: Desclée de Brouwer.

Tenorio, M., Arango, P., Aparicio,A., Benavente, C., Thibaut., C., \& Rosas, R. (2012). Test de Evaluación Neuropsicológica Infantil Teni. Santiago de Chile: CedeTi UC.

Van der Ven, S., Kroesbergen, E., Boom, J., \& Leseman, P. (2013). The structure of executive functions in children: A closer examination of inhibition, shifting, and updating. British Journal of Developmental Psychology, 31(1), 70-87. doi:10.1111/ j.2044-835X.2012.02079.x

Vargas-Rubilar, J., \& Arán-Filippetti, V. (2014). Importancia de la parentalidad para el desarrollo cognitivo infantil: una revisión teórica. Revista Latinoamericana de Ciencias Sociales, Niñez y Juventud, 12(1), 171-186. doi:10.11600/169271 $5 \mathrm{x} .1219110813$

Vygotsky, L. (1978). Mind in society: The development of higher psychological 
processes. Cambridge: Harvard University Press.

Weiss, H. (2014). Nuevas orientaciones sobre el involucramiento familiar en el aprendizaje. Santiago de Chile: Fundación CAP.

Zambrano, J. (2013). Análisis multinivel del rendimiento escolar en matemáticas para cuarto grado de educación básica primaria en Colombia. Sociedad y Economía, (25), 205-235.

Zelazo, P., \& Müller, U. (2002). Executive function in typical and atypical development. En U. Goswami (Ed.), Handbook of childhood cognitive development (pp.445-469). Oxford: Blackwell. doi:10.1002/9780470996652. $\operatorname{ch} 20$ 